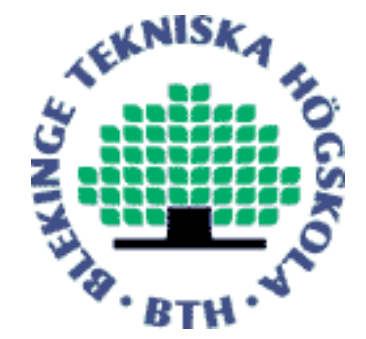

Copyright (C) 2012 IEEE.

Citation for the published paper:

Simulations of the VISIR Open Lab Platform

Mikael Swartling, Josef Ström Bartunek, Kristian Nilsson, Ingvar Gustavsson, Markus Fiedler

Remote Engineering and Virtual Instrumentation

2012 Bilbao, Spain

This material is posted here with permission of the IEEE. Such permission of the IEEE does not in any way imply IEEE endorsement of any of BTH's products or services Internal or personal use of this material is permitted. However, permission to reprint/republish this material for advertising or promotional purposes or for creating new collective works for resale or redistribution must be obtained from the IEEE by sending a blank email message to pubs-permissions@iee.org.

By choosing to view this document, you agree to all provisions of the copyright laws protecting it. 


\title{
Simulations of the VISIR Open Lab Platform
}

\author{
Mikael Swartling*, Josef Ström Bartůněk*, Kristian Nilsson*, Ingvar Gustavsson* and Markus Fiedler ${ }^{\dagger}$ \\ ${ }^{*}$ Department of Electrical Engineering \\ † Department of Computing \\ Blekinge Institute of Technology, SE-371 79 Karlskrona, Sweden
}

\begin{abstract}
This paper presents a queue simulation of a remote laboratory based on the VISIR Open Lab Platform designed at Blekinge Institute of Technology. A model of this VISIR laboratory and statistical distributions of how users interact with the system based on real log files are presented. The system is then simulated in order to determine how many concurrent students that can be allowed to use the laboratory while at the same time keeping a low response time to ensure the quality of the service. The results show, in a worst case setup with approximately $300 \mathrm{~ms}$ response time per experiment, that roughly 100 concurrent users is an upper limit to ensure an average response time below $2 \mathrm{~s}$. The results also show that raising the limit of the desired experiment response time does not necessarily increase the number allowed concurrent users significantly once the system is saturated. However, improving the experiment response time can significantly increase the number of users that can simultaneously be connected.

Index Terms-VISIR, Simulation, Quality of Experience
\end{abstract}

\section{INTRODUCTION}

Instructional laboratories for electrical experiments are widely used in the educational system because both majors and non-majors in electrical engineering perform electrical experiments. Most universities around the world providing engineering education also have such laboratories and they often look the same; they are a kind of de facto standard well-known among teachers and students. Such laboratories contain a number of identical workbenches consists of at least a DC power supply, a function generator, a digital multi-meter (DMM), an oscilloscope and a breadboard.

The VISIR Open Lab Platform designed at the Department of Electrical Engineering at Blekinge Institute of Technology (BTH) in Sweden is an architecture to provide remote access to instructional laboratories [1], [2]. In VISIR laboratories, students perform physical experiments and laboratory work remotely over the Internet. The remote laboratory project started as a feasibility study in 1999 in order to provide more opportunities for students to conduct electrical experiments. Traditional circuit theory experiments have been conducted over the Internet using an online workbench at BTH from different locations simultaneously [3], [4]. VISIR laboratoriees each containing one online workbench have been set up at a number of universities worldwide [5]-[7] and the development of a grid laboratory that will use the resources more efficiently is in progress [8].

Opening a hands-on laboratory for remote access means providing a computer-mediated laboratory [9]. The student connecting to a VISIR laboratory remotely wires the circuit and connects instruments on a virtual breadboard displayed on the computer screen. A relay switching matrix then connects the physical components and instruments [10]. The VISIR Open Lab Platform specifies a few distinct parts. A measurement server executes requests from an experiment client. An equipment server that hosts and controls the instruments and the switching matrix. Finally, an experiment client written in Adobe Flash is embedded in an HTML page served by the web interface. A web interface handles administration, user admission and resource scheduling. A simplified schematic view of the platform is shown in figure 1.

In figure 1, the users interact with the public interface and experiment requests are submitted to the measurement and the equipment servers via the internal interface. Only a single experiment can be performed by the measurement and the equipment servers at any given time. Multiple submitted experiment requests must therefore be queued and handled sequentially. The public interface is currently limited to 16 concurrent users experimenting to ensure that the response time for a submitted experiment is low so that a sufficient quality of experience is ensured.

Figure 2 shows a typical matrix used in a VISIR laboratory at BTH and is the target of the simulations performed in this paper. The figure shows, from the bottom, a source board, two instrument boards and a component board stacked [10]. Additional instrument and component boards can be stacked as required by particular experiments or setups. The current desired goal with this VISIR laboratory is that a submitted experiment request shall be performed and that the result shall be returned and presented to the user within two seconds. All the simulations performed in this paper will therefore be compared against this desired two-second limit.

\section{Goal of the Simulations}

The goal of this paper is to present and evaluate a model of the response times the users experience by simulating the activity and to examine how the number of connected users affects the response time. The simulations are performed to determine if the current limit of 16 concurrent users can be raised and at what point the quality of service (as determined by the two-second response time) can be compromised.

\section{Simulation Model and Parameters}

\section{A. Simulation Model}

The queue system model consists of a fixed number of independent users submitting experiments and a single server 


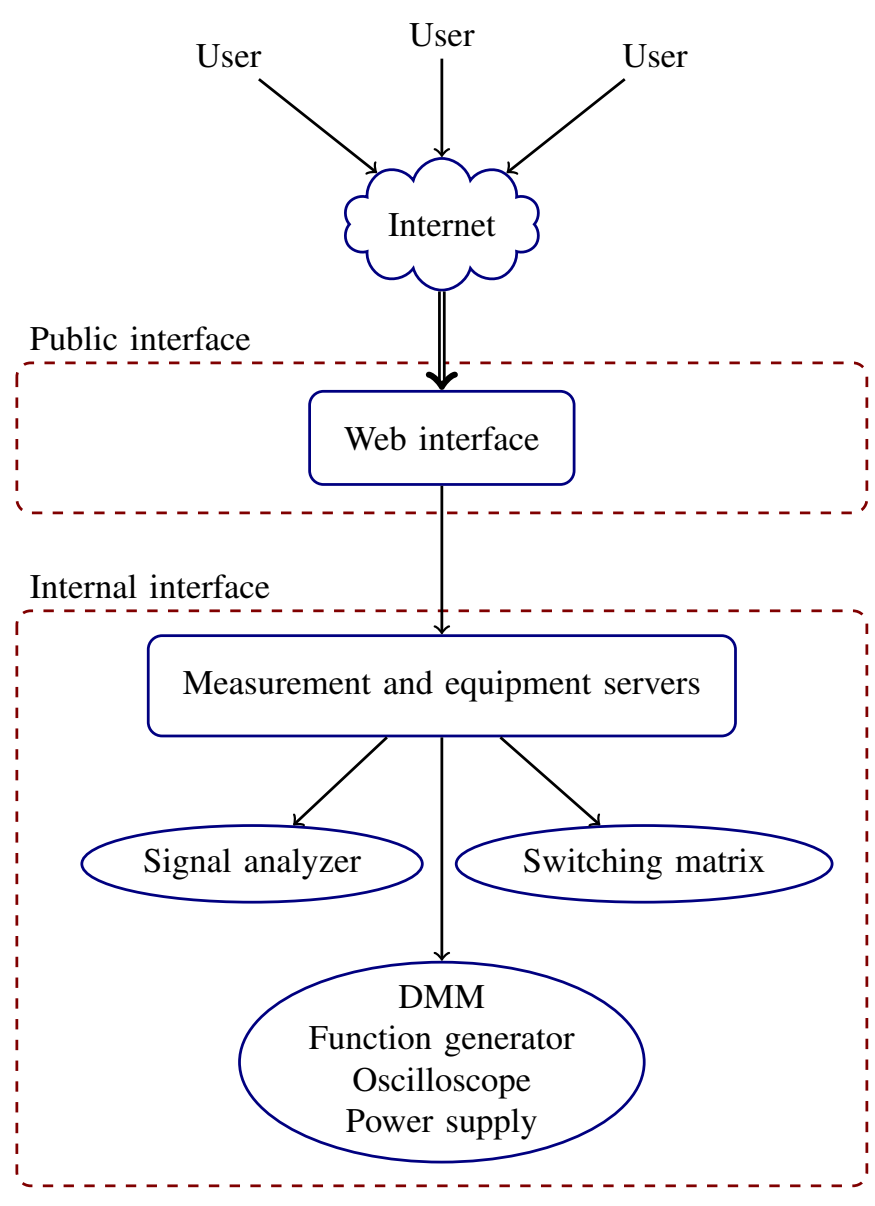

Fig. 1. A simplified schematic view of the VISIR Open Lab Platform.

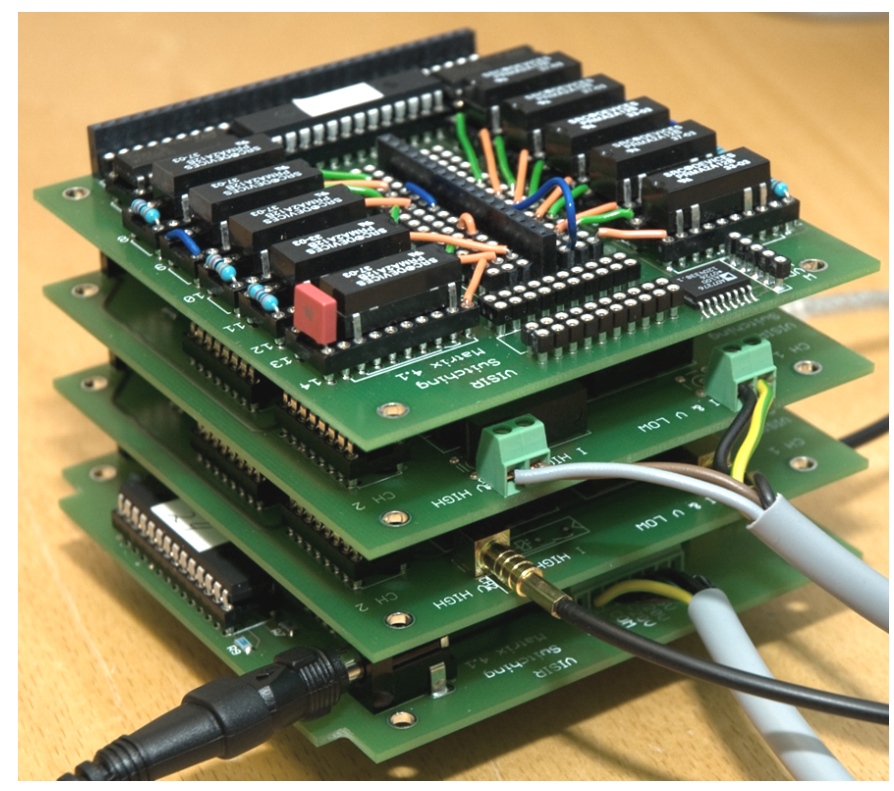

Fig. 2. A switching matrix consisting of (from the bottom) a source board, two instrument boards and a component board stacked. handling the experiments. If the server is busy performing an experiment, then new submissions are placed in a queue and served on a first-come first-serve basis. The goal is to analyze how the submission queue behaves to allow as many concurrent users as possible while at the same time having a short submission queue time. In the particular setup being analyzed, the response time for an experiment (from when the experiment is submitted to when the result is presented to the user) is required by choice to be less than two seconds to ensure the quality of the experience for the users.

The arrival of experiments from the connected users is a closed system where a limited number of users submit new experiments. A user cannot submit a new experiment until the previous experiment has been finished and the result has been presented. The submission queue is thus strictly limited to one less than the total number of user in the system. Therefore, there is a guaranteed upper limit on the experiment service time:

$$
T_{\mathrm{s}} \leq N \cdot T_{\mathrm{e}} .
$$

Here, $T_{\mathrm{s}}$ is the total time to service an experiment including queue time, $T_{\mathrm{e}}$ is the experiment time which is the time to perform a single experiment and $N$ is the number of connected users. The experiment time $T_{e}$ is typically not constant but depends on what experiment a user is performing. Given the two-second upper limit on the service time $T_{s}$, the number of users $N$ can, therefore, not be calculated directly other than ensuring that the maximum desired service time in the worst case does not exceed the desired limit.

Parameters that have to be identified for the simulations are:

- the experiment arrival distribution to represent the rate at which a user submits new experiments,

- the number of concurrent users to see how the system handles an increasing number of users, and

- the experiment time to represent the time taken to perform a submitted experiment.

The only parameter that has to be estimated is the experiment arrival distribution. The number of concurrent users is a controlled parameter for each simulation in order to determine a saturation point for the system and the experiment time is taken from timing diagrams and data sheets for the system components. Log files from real experiment servers are used to estimate the experiment arrival distribution.

\section{B. Experiment Arrival Distribution}

When parsing the log files containing activities from multiple users, the individual users must be identified and separated to determine how a single user behaves on average so that the number of users and the behavior of the users can be controlled individually in the simulations. Assume that $t_{u}(n)$ is the time at which the user $u$ submitted the experiment $n$ and that $\Delta t_{u}(n)=t_{u}(n+1)-t_{u}(n)$ is the time between experiment $n$ and $n+1$ for the user $u$. Thus, $\Delta t_{u}(n)$ is a sample of the stochastic process that is the experiment arrival distribution. Furthermore, the set $\mathbf{P}$ contains all sample point pairs $\{u, n\}$. 


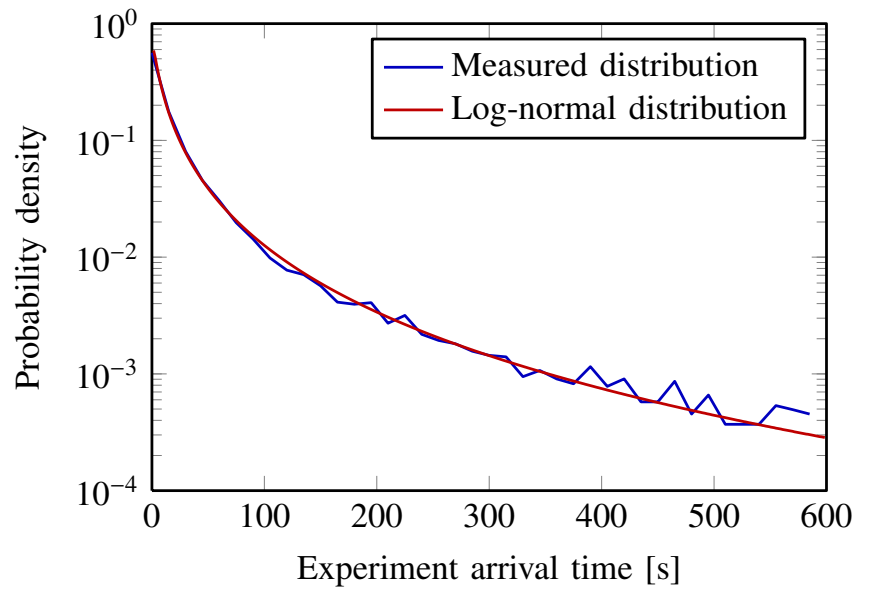

Fig. 3. The experiment arrival times modeled as a log-normal distribution with $\mu=33 \mathrm{~s}$ and $\sigma=65 \mathrm{~s}$.

The mean $\mu$ and variance $\sigma^{2}$ of the experiment arrival times are then estimated as

$$
\begin{aligned}
\mu & =\frac{1}{|\mathbf{P}|} \sum_{\{u, n\}} \Delta t_{u}(n) \\
\sigma^{2} & =\frac{1}{|\mathbf{P}|-1} \sum_{\{u, n\}}\left(\Delta t_{u}(n)-\mu\right)^{2} .
\end{aligned}
$$

By matching usage patterns for various distributions and empirically studying the actual distribution of the available data, the experiment arrival times are assumed to be lognormally distributed. Given the mean and the variance of the distribution, the parameters $\mu_{\mathrm{LN}}$ and $\sigma_{\mathrm{LN}}^{2}$ for the log-normal distribution is calculated as [11]

$$
\begin{gathered}
\mu_{\mathrm{LN}}=\log \frac{\mu^{2}}{\sqrt{\sigma^{2}+\mu^{2}}} \\
\sigma_{\mathrm{LN}}^{2}=\log \left(\frac{\sigma^{2}}{\mu^{2}}+1\right)
\end{gathered}
$$

and the modeled experiment arrival time is

$$
\Delta t(n) \sim \exp \left\{\mathcal{N}\left(\mu_{\mathrm{LN}}, \sigma_{\mathrm{LN}}^{2}\right)\right\} .
$$

The parameters $\mu_{\mathrm{LN}}$ and $\sigma_{\mathrm{LN}}$ are the mean and variance of the normal distribution used to generate the log-normal distribution. Figure 3 shows the probability density function of the measured experiment arrival times and the corresponding estimated log-normal distribution.

\section{Experiment Time}

The experiment time is the time taken to perform a single submitted experiment, to present the result to the user and to initiate the next experiment in the service queue. However, to derive the service time, the service time is split into three parts:

- Warm-up time, $T_{\mathrm{w}}$, models the time to start up an experiment. For example, the time to configure the voltage or signal generator, multi-meter, oscilloscope or spectrum analyzer, and switch relays in the component matrix.
TABLE I

THE TIMES IN MILLISECONDS FOR CONFIGURING THE EQUIPMENT AND FOR MEASURING AN EXPERIMENT.

\begin{tabular}{lccc}
\hline Unit & $T_{\mathrm{w}}[\mathrm{ms}]$ & $T_{\mathrm{m}}[\mathrm{ms}]$ & $T_{\mathrm{c}}[\mathrm{ms}]$ \\
\hline General overhead & 10 & 10 \\
Matrix switch (per layer) & 0.08 & & 0.08 \\
Power supply +6 V & 1 & & 25 \\
Power supply $\pm 20 \mathrm{~V}$ & 1 & & 56 \\
Function generator & 1 & 5 & 1 \\
DMM, auto range DC & & 250 & \\
DMM, auto range AC & & 50 & \\
DMM, auto range resistance & \multicolumn{3}{c}{50} \\
Oscilloscope & \multicolumn{3}{c}{10} \\
Measurement & &
\end{tabular}

- Measurement time, $T_{\mathrm{m}}$, models the time taken to perform the actual experiment. For example, the time for the circuit to stabilize and the time to perform a measurement using some of the attached equipments.

- Cool-down time, $T_{\mathrm{c}}$, models the time to shut down the experiment. For example, the time to read back and present the results of the experiment to the user.

The experiment time is then

$$
T_{\mathrm{e}}=T_{\mathrm{w}}+T_{\mathrm{m}}+T_{\mathrm{c}} .
$$

The warm-up, the measurement and the cool-down times are determined from data sheets, by timing the circuits and from fixed times to have the circuit stabilize itself for a reliable measurement.

The times for the different units to perform an experiment are shown in table I and table II lists the actual instruments used for the physical experiments in this particular setup. The warm-up and cool-down times are obtained from the data sheets for the equipments used in the experiment setup. The warm-up time is to set up the power supply and to switch the component matrix. The measurement time is a fixed time of $10 \mathrm{~ms}$ to stabilize the circuit and an additional measurement time depending on the equipments and the configuration used for the measurement. The cool-down time is to let the power supply shut down and to reset the component matrix. The component matrix typically consists of 8 to 12 layers.

Not all combinations of equipments and measurements are relevant to the analysis. For example, the function generator, oscilloscope and AC auto-ranging are not always relevant for DC circuits, the DC power supply is not always relevant for AC circuits, and neither the power supply nor the function generator are relevant for resistance circuits. The analysis will focus on the worst case configuration, which means an $\mathrm{AC}$ circuit, to ensure that the simulations represent the behavior under maximum queue times.

\section{Simulation and Results}

The main goal is to ensure that the users are served rapidly when performing an experiment. In the worst case, the maximum number of concurrent users becomes

$$
N_{\max }=\left\lfloor\frac{T_{\mathrm{d}}}{T_{\mathrm{e}}}\right\rfloor
$$


TABLE II

INSTRUMENTS USED FOR THE DIFFERENT UNITS OF THE PHYSICAL COMPONENT MATRIX.

\begin{tabular}{ll}
\hline Unit & Instrument \\
\hline Power supply & NI-PxI-4110 \\
Function generator & NI-PxI-5402 \\
Digital multi-meter & NI-PxI-4060 \\
Oscilloscope & NI-PxI-5112 \\
\hline
\end{tabular}

where $T_{\mathrm{d}}$ is the desired maximum service time. The worst case is when all $N_{\max }$ users submit an experiment at the same time: the last user in the queue is still guaranteed to be served within $T_{\mathrm{d}}$. Instead of enforcing a worst case limit, simulations are performed to determine a limit on the number of users that still provides a low service time to some probability. The simulations are performed using the user model, the equipment model and their parameters described in the previous section. A worst case setup is assumed: an AC circuit with DMM autoranging. The set times are therefore $T_{\mathrm{w}}=11 \mathrm{~ms}, T_{\mathrm{m}}=260 \mathrm{~ms}$ and $T_{\mathrm{c}}=11 \mathrm{~ms}$. Thus, the experiment response time is $T_{\mathrm{e}}=$ $282 \mathrm{~ms}$.

The simulations are performed with 10000000 experiments per batch, corresponding to approximately 40 days of continuous load from 100 concurrent users according to the estimated distribution. Furthermore, the results are the average of 10 batches. The confidence intervals for the estimated values are very small due to the large number of experiments being simulated. The primary goal of the simulations is not to estimate precise values, but to determine general trends to assist in determining a limit on how many users that can be connected and perform experiments at the same time. For this reason, confidence intervals have been omitted since even a high confidence interval is not large enough to significantly affect any decision on limiting the number of users.

\section{A. Simulation: Wait Times}

Figure 4 shows the result from the simulation of the time a user has to wait for an experiment to complete. As the number of concurrent users increases, the wait time also increases since there will also be an increasing number of queued experiment requests that have to be served. The figure shows three graphs: the average wait time per user, the maximum wait time for any user encountered during the simulation and the limit of the maximum possible wait time a user can theoretically experience. The figure also shows the desired upper limit of the response time for reference which is $T_{\mathrm{d}}=2 \mathrm{~s}$. The maximum wait time during a simulation depends on how long the simulation is performed and it will increase towards the theoretical limit as the simulation time approaches infinity. The limit of the maximum possible wait time occurs if all users submit an experiment at the same time and is simply evaluated as $T_{\max }=N \cdot T_{\mathrm{e}}$.

To guarantee a service time below $T_{\mathrm{d}}$, the maximum number of users can be calculated from (8). The maximum number of users can also be observed from figure 4 where the graph for $T_{\max }$ intersects the desired upper limit. The number of concurrent users can be greatly increased if the demand for a desired limit is relaxed to allow, on average, a service time below the desired limit; from approximately 10 users for the theoretical limit to around 100 users for the average wait time. Even if the maximum wait time for any single user is considered, 30 to 40 concurrent users may be allowed. When the simulated system reaches a breaking point at around 100 users for the average case, the wait time increases at a rate similar to the theoretical limit.

An additional observation is that raising the desired service time limit $T_{\mathrm{d}}$ does not significantly change the number of allowed users after the breaking point around 100 users. As seen from figure 4 , the limit in the average case is approximately 110 users. However, doubling the limit to $T_{\mathrm{d}}=4 \mathrm{~s}$ only increases the number of allowed users to approximately 120 .

\section{B. Simulation: Queued Users}

Figure 5 shows the estimated probability that a submitted experiment is queued. As the number of concurrent users increases, the chance of a submitted experiment being queued is increased. The figure shows the estimation of the probability that an experiment is not queued at all, is queued for shorter than the desired limit and is queued for longer than the the desired limit. The time an experiment is waiting in the queue is $T_{\mathrm{q}}$.

Before the system starts to saturate around 100 users, the chance that an experiment is queued increases linearly. These queued submissions are, however, queued for a time less than the desired limit and are thus not a problem for the quality of the service. Beyond the saturation point, the probability of being queued for longer than the desired limit increase dramatically. Even though nearly half of the experiments are queued with 50 to 60 concurrent users, almost none of them are queued longer than the desired limit.

\section{Simulation: Improved Experiment Response Time}

The previously presented simulations were performed with a worst case setup. It was there shown that the number of allowed users cannot be significantly improved by allowing a slightly longer desired maximum response time. Figure 6 shows the average wait time per user for different experiment times. The figure shows that the average wait time can be significantly decreased when the experiment time is improved. This corresponds to performing experiments using equipments with a lower response time, for example due performing other types of measurements (DC vs. AC measurements), or simply due to better performing instruments. Likewise, increasing the experiment time increases the average wait time.

\section{Conclusions}

The VISIR Open Lab Platform was analyzed and simulated to determine how the response times behave when different number of users interact with it. The goal was to determine how many users can be allowed to log in and experiment at the same time while at the same time ensuring a low service time. From a model of the queuing system, distributions 


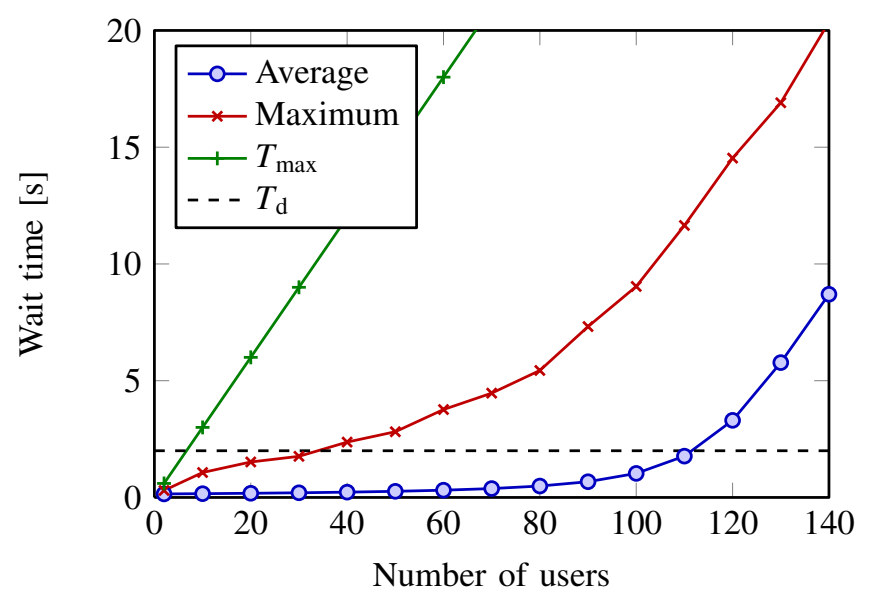

Fig. 4. The estimated wait times for a submitted experiment when the number of users increases.

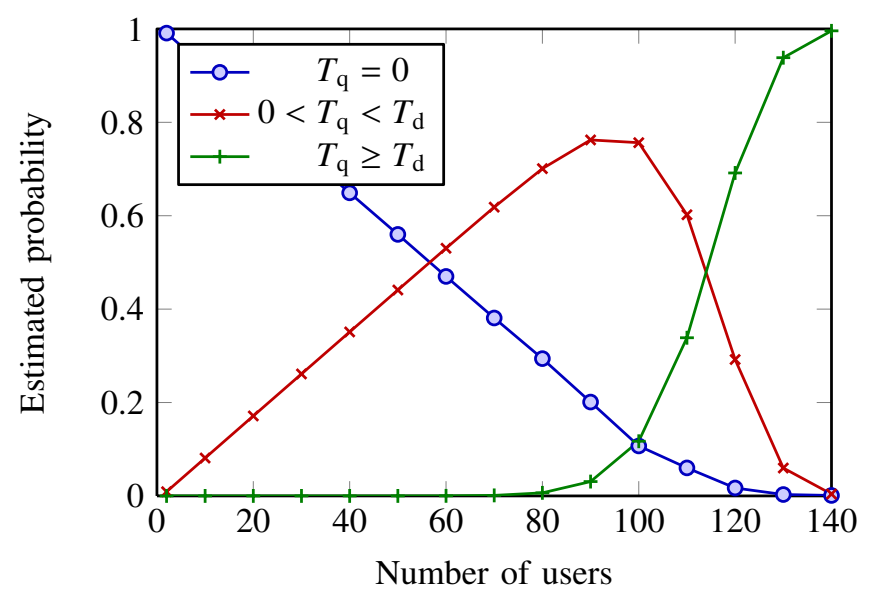

Fig. 5. The estimated probability that a submitted experiment is queued when the number of users increases.

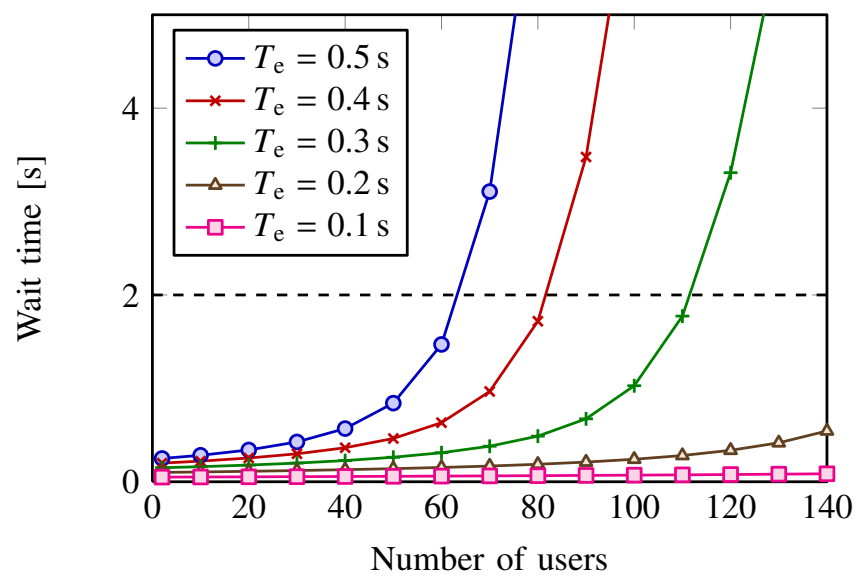

Fig. 6. The estimated wait times for a submitted experiment for different experiment times when the number of users increases. and parameters of how users perform experiments and the behavior of the physical equipment it can be seen that even with around 100 users the response time is still acceptable on average. Occasional wait times above the desired twosecond limit may be allowed to be able to increase the number of concurrent users. The simulations were performed with a worst case setup. It was, furthermore, shown that improving the experiment time can significantly improve the performance of the system, which in turn allows for more users.

Considering the number of students of the classes performing the experiments during a course, this limit may not be a practical problem. All students may, of course, not be connected at the same time during a course. However, the planning of the course may naturally force the students to perform the experiments on given times during the course. The number of concurrent users was not so much of a practical problem with respect to response times. Simulations of the system show that the limit on the number of concurrent users can be greatly increased and still provide a low service time.

\section{REFERENCES}

[1] J. Zackrisson, I. Gustavsson, and L. Håkansson, "An overview of the VISIR open source software distribution 2007," in Proceedings of the 2007 REV Conference, Jun. 2007.

[2] [Online]. Available: http://openlabs.bth.se/

[3] I. Gustavsson, "A remote access laboratory for electrical circuit experiments," International Journal of Engineering Education, vol. 19, 2003.

[4] I. Gustavsson, J. Zackrisson, L. Håkansson, I. Claesson, and T. Lagö, "The VISIR project—an open source software initiative for distributed online laboratories," in Proceedings of the 2007 REV Conference, Jun. 2007.

[5] J. Garcia-Zubia, I. Gustavsson, U. Hernandez-Jayo, P. Orduna, I. Angulo, L. Rodriguez, and D. L. de Ipina, "Using VISIR: Experiments, subjects and students," International Journal of Online Engineering, vol. 7, no. S2, pp. 11-14, 2011.

[6] D. G. Zutin, "A VISIR lab server for the iLab shared architecture," International Journal of Online Engineering, vol. 7, no. S1, pp. 14-17, 2011.

[7] G. Alves, M. Marques, C. Viegas, M. Costa Lobo, R. Barral, R. Couto, F. Jacob, C. Ramos, G. Vilao, D. Covita, J. Alves, P. Guimaraes, and I. Gustavsson, "Using VISIR in a large undergraduate course: Preliminary assessment results," in IEEE Global Engineering Education Conference, 2011, pp. 1125-1132.

[8] I. Gustavsson, J. Zackrisson, K. Nilsson, J. Garcia-Zubia, L. Håkansson, I. Claesson, and T. Lagö, "A flexible instructional electronics laboratory with local and remote lab workbenches in a grid," International Journal of Online Engineering, vol. 4, no. 2, 2008.

[9] L. Gomes and J. Garcia-Zubia, Eds., Advances on Remote Laboratories and e-Learning Experiences. University of Deusto, Bilbao, Spain, 2007.

[10] I. Gustavsson, J. Zackrisson, J. S. Bartůněk, K. Nilsson, L. Håkansson, I. Claesson, and T. Lagö, "Telemanipulator for remote wiring of electrical circuits," in Proceedings of the 2008 REV Conference, Jun. 2008.

[11] A. M. Law, Simulation Modeling and Analysis, 4th ed. McGraw-Hill, 2007. 